\title{
Research on New Methods of Multi-project Based on Entropy and Particle Swarm Optimization for Resource Leveling Problem
}

\author{
Wen-min HAN ${ }^{1,}$ a), Bin-bin ZHU ${ }^{2, \text { b) }}$ \\ ${ }^{1}$ School of Economic and Management, Jiang su University of Science and Technology, Zhen \\ jiang 212003, China; \\ ${ }^{2}$ School of Civil Engineering and Architecture, Jiang su University of Science and Technology, \\ Zhen jiang 212003, China; \\ b) Corresponding author: 8196972636@qq.com
}

\begin{abstract}
Key words: project management; multiple resources leveling optimization; resource entropy Abstract: The resource leveling optimization is an important content of project management, and the optimization of resource leveling are helpful to solve the contradiction between the requirements and supply, which is of significant importance to the project management. The existing research focus on the innovation of the method, and the research on the innovation of the resource equilibrium model are relatively few. Therefore, in this paper, a resource leveling optimization model of multi-project based on entropy function is designed. Simultaneously, genetic algorithm is adopted to solve this problem.
\end{abstract}

\section{Introduction}

Resource equilibrium problem is an optimization problem with important economic significance. In traditional project construction management practice ${ }^{[1]}$, the resource equilibrium problem was rarely researched and most literature were focused on construction period, quality, cost and other relevant objectives ${ }^{[2]}$.

Along with the enlargement and complication of the construction projects, stricter requirements have been proposed for the resource allocation in the project construction management process ${ }^{[3]}$. A task process usually needs to consume multiple resources at different amounts, so it is necessary to equalize and optimize multiple resources. However, the existing research is mainly focused on discussing method innovation but the resource equilibrium model is rarely innovative researched ${ }^{[4]}$. Along with the development of simulation algorithms especially in recent years, more and more scholars start concerning the application of the intelligent algorithms in resource optimization, and try to apply the intelligent algorithms to solve the old resource equilibrium models ${ }^{[5]}$. For example, Q I J et al. ${ }^{[5]}$ proposed a new accurate solution for the multi-project resource equilibrium problem on the basis of the genetic algorithm principle; $\mathrm{M} \mathrm{N}$ et al. ${ }^{[6]}$ researched the application of the genetic algorithm based on binary coding for solving the resource equilibrium problem in network resource optimization; $\mathrm{Li} \mathrm{H} \mathrm{B} \mathrm{et} \mathrm{al.}{ }^{[7]}$ proposed an improved least moment method for multiple resources equilibrium optimization and obtained a result superior to that obtained from the traditional method.

According to above research and analysis, the existing resource equilibrium research is mainly based on the traditional network planning technique, such as critical path method and PERT ${ }^{[8]}$, and is mainly focused on the application of the intelligent algorithms, but the model improvement is rarely researched. In recent years, some scholars start discussing the resource equilibrium model problem. For example, He L et al. ${ }^{[9]}$ defined the resource entropy concept and adopted the entropy function to establish the resource equilibrium optimization model for project management; Tang Y, Liu R, Sun $Q^{[10]}$ researched the multiple resources equilibrium problem under the supporting construction conditions, and introduced the resource entropy for resource equilibrium measurement, as well as established the multiple resources equilibrium optimization model considering the supporting construction ${ }^{[11]}$.

Firstly, relevant knowledge of resource equilibrium was introduced in this paper, the 
multi-project multiple resources equilibrium problem based on critical chain technique was discussed, and relevant resource entropy theory was combined to establish the corresponding optimization model; then, the genetic algorithm was adopted to discuss the application thereof in the model. Specially, the condition of multiple resources in the multi-project was considered in this paper, and the analytic hierarchy process was adopted for multiple resources to determine the weight value of each resource, and the resource entropy was adopted as the uniform dimension of various resources for solving the multiple resources equilibrium problem.

\section{Entropy Weight Model for Multiple Resources of Multi-project Equilibrium Problem}

2.1 Resource Equilibrium Problem Analysis. In practical project construction management process, the resource equilibrium problem is a typical and significant optimization problem ${ }^{[12]}$. The resource equilibrium problem of an engineering project generally means the equilibrium consumption of one or multiple resources within the construction period through adjusting the actual start time of the activity process in the project. This paper is mainly focused on the problem of multi-resource equilibrium under multiple project parallel condition ${ }^{[13]}$. At present, the multiple resource identical method is mainly adopted for the research in order to convert multiple resources equilibrium into single-resource equilibrium ${ }^{[14]}$. For simplification, the traditional multiple resources equilibrium is usually converted into single-resource equilibrium for solution. But the result obtained thereby should be further determined, because the equivalent requirement of the resources cannot reflect the equilibrium degree of the actual consumption of each resource. The equivalent requirement curve seemingly equalized may be engaged by the disequilibrium consumption curves of multiple resources.

Therefore, in allusion to the resource equilibrium engagement problem, both the sub-project resource equilibrium and the multi-project overall resource equilibrium were considered in this paper at the same time, and the multiple objective model was converted into the single-objective model for multiple resource equilibrium. Meanwhile, in order to better represent the equilibrium degree of resource utilization and eliminate the influence of different resource dimensions, the resource entropy was introduced as the resource equilibrium measurement in this paper.

2.2 Objective Function. In this paper, the corresponding resource entropy was respectively set for each resource in the multi-project and the sub-projects for resource equilibrium optimization. According to above analysis of sub-project resource equilibrium and multi-project resource equilibrium, the model of multi-project resource equilibrium was established in this paper as follows:

$$
M A X . E V=\lambda_{1} * \sum_{w=1}^{W} c_{w} * E V_{K}^{w}+\lambda_{2} * \frac{1}{N} * \sum_{w=1}^{W} c_{w} * \sum_{l=1}^{N} E V_{l}^{w}
$$

In the formula:

$$
\begin{aligned}
& E V_{K}^{m}=\sum_{\mathrm{t}=1}^{T}\left[\left\{R^{m}(t) / \sum_{t=1}^{T} R^{m}(t)\right\} * \ln \left\{R^{m}(t) / \sum_{t=1}^{T} R^{m}(t)\right\}\right] \\
& E V_{l}^{w}=\sum_{\mathrm{t}=1}^{T_{l}}\left[\left\{R_{l}^{w}(t) / \sum_{t=1}^{T_{l}} R_{l}^{w}(t)\right\} * \ln \left\{R_{l}^{w}(t) / \sum_{t=1}^{T_{t}} R_{l}^{w}(t)\right\}\right]
\end{aligned}
$$

$E V_{k}^{w}$ : It represents the resource entropy of the resource $w$ used by all activities of the multi-project whole within the duration of the total construction;

$E V_{l}^{w}$ : It represents the resource entropy of the resource ${ }^{w}$ used by all activities of the sub-projects within the construction duration;

$\lambda_{1}, \lambda_{2}$ : They respectively represent the proportions of the multi-project equilibrium and the sum of the local equilibrium, wherein $\lambda_{1}+\lambda_{2}=1$;

$w$ : It represents multi-project resource types $((1 \leq w \leq W))$; 
$c_{w}$ : It represents the weight of the ${ }^{w}$ resource.

\subsection{Constraint Condition Analysis}

2.3.1 Float Constraints. The activities are limited by the total time difference in the resource equilibrium optimization process, so the actual start time of each activity must meet the following condition:

In the formula:

$$
\operatorname{Es}\left(i_{l}\right) \leq T s\left(i_{l}\right) \leq \operatorname{Ls}\left(i_{l}\right), \quad i_{l} \in E_{l}, l=1,2 \ldots N
$$

$E s\left(i_{l}\right)$ : The earliest start time of activity ${ }^{i_{l}}$;

$L s\left(i_{l}\right)$ : The latest start time of activity ${ }^{i_{l}}$;

$T_{s}\left(i_{l}\right)$ : The actual start time of activity ${ }^{i_{l}}$;

$E_{l}$ : The set of all activities in sub-project $l(l=1,2 \ldots N)$.

2.3.2 Adjacent Relationship Constraints In a multi-project activity, the actual start time of any activity should not be earlier than the actual end time of any previous adjacent activity:

$$
\max \left(T s\left(j_{l}\right)+d\left(j_{l}\right)\right) \leq T s\left(i_{l}\right) \leq L s\left(i_{l}\right), \forall j_{l} \in V\left(i_{l}\right) ;
$$

In the formula:

$V\left(i_{l}\right)$ : The set of all previous adjacent activities of activity ${ }^{i_{l}}$;

$d\left(j_{l}\right)$ : Construction period of activity ${ }^{i_{l}}$.

2.3.3 Resource Constraints. In the multi-project resource utilization process and the local resource utilization process, the usage amount of the resources in a certain period must be more than the supply amount of the resources, so the resource consumption should meet the following condition.

$$
\begin{aligned}
& \sum_{i_{i} \in E_{l}} R_{t}^{w}\left(i_{l}\right)=R_{l}^{w}(t) ; \quad u_{l, t}^{w} \geq R_{l}^{w}(t) \\
& \sum_{l=1}^{N} R_{l}^{w}(t)=\sum_{l=1}^{N} \sum_{i_{l} \in E_{l}} R_{t}^{w}\left(i_{l}\right)=R^{w}(t) ; \quad u_{t}^{w} \geq R^{w}(t) ; \\
& R_{t}^{w}\left(i_{l}\right)=\left\{\begin{array}{l}
R^{w}\left(i_{l}\right), T s\left(i_{l}\right) \leq t \leq f\left(i_{l}\right) ; \\
0, \text { else }
\end{array}\right.
\end{aligned}
$$

In the formula:

$R_{t}^{w}\left(i_{l}\right)$ : The resource ${ }^{w}$ consumption of activity ${ }^{i}$ in sub-project $l^{l}$ at the ${ }^{t}$ moment;

$R\left(i_{l}\right)$ : The resource ${ }^{w}$ consumption of activity ${ }^{i}$ in sub-project ${ }^{l}$ within the unit time;

$R^{w}(t)$ : The total resource ${ }^{w}$ consumption of all activities in the multi-project at the ${ }^{t}$ moment;

$R_{l}^{w}(t)$ : The total resource ${ }^{w}$ consumption of all activities in sub-project ${ }^{l}$ at the ${ }^{t}$ moment;

$u_{t}^{w}:$ The maximum supply amount of the resource ${ }^{w}$ at the ${ }^{t}$ moment;

$u_{l, t}^{w}$ : The maximum supply amount of the resource ${ }^{w}$ in sub-project $l$ at the ${ }^{t}$ moment;

$f\left(i_{l}\right)$ : The actual end time of activity ${ }_{l}$.

\subsection{Genetic Algorithm}

2.4.1 Design of Code. In this paper, the actual start time $T s\left(i_{l}\right)$ of the activity $i_{l}$ is encoded as a chromosome. The specific chromosome structure is shown in figure 1.

\begin{tabular}{|l|l|l|l|l|l|l|l|l|l|}
\hline $\mathrm{Ts}(\mathrm{A} 1)$ & $\mathrm{Ts}(\mathrm{B} 1)$ & $\cdots$ & $\mathrm{Ts}(\mathrm{N} 1)$ & $\cdots$ & $\mathrm{Ts}(\mathrm{An})$ & $\mathrm{Ts}(\mathrm{Bn})$ & $\mathrm{Ts}(\mathrm{Cn})$ & $\cdots$ & $\mathrm{Ts}(\mathrm{Nn})$ \\
\hline
\end{tabular}

Fig.1 Design of Code

2.4.2 The Analysis of Constraints. In dealing with the constraints all into the following forms: 


$$
0 \leq T s\left(i_{l}\right)-E s\left(i_{l}\right) \leq E\left(i_{l}\right)
$$

2.4.3 Fitness Function. The fitness function value of individual $\mathrm{c}$ is shown below.

$$
F\left(v_{k}\right)=M+E V\left(v_{k}\right)-\alpha f\left(v_{k}\right)
$$

$v_{k}$ is the $k$ chromosome of the current population, $E V\left(v_{k}\right)$ is the objective function value, and $\mathrm{M}$ is a positive constant, in order to ensure the fitness function value, $\alpha$ is the penalty factor, $f\left(v_{k}\right)$ is the penalty function.

2.4.4 Evolutionary Strategy. (1) Selecting the operator, and calculate the fitness of all the individuals in current population, and the rotary wheel method is adopted for selection of individual.

(2) The single-point method is adopted for cross and variation;

(3) The maximum generation number and the maximum resource entropy have been generated as the termination condition.

\section{Calculation Case Verification}

In order to verify the validity of the proposed indexes and the multiple-project robustness objective model, this article adopts the parallel multiple-project case composed with 3 projects.

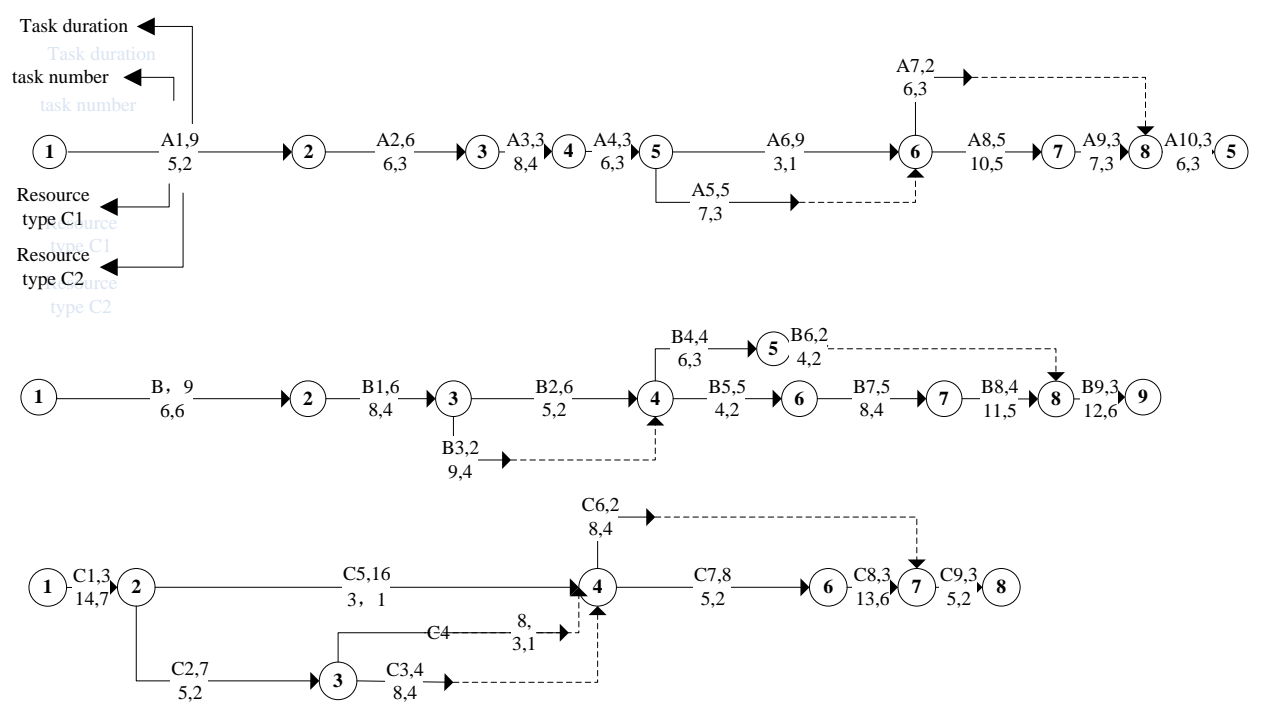

$\begin{array}{lllllllllllllllllllllllllllllllllllllllllllllll}0 & 1 & 2 & 3 & 4 & 5 & 6 & 7 & 8 & 9 & 1 & 1 & 1 & 1 & 1 & 1 & 1 & 1 & 1 & 1 & 2 & 2 & 2 & 2 & 2 & 2 & 2 & 2 & 2 & 2 & 3 & 3 & 3 & 3 & 3 & 3 & 3 & 3 & 3 & 3 & 4 & 4 & 4 & 4 & 4 & 4 \\ 0 & 0 & 1 & 2 & 3 & 4 & 5 & 6 & 7 & 8 & 9 & 0 & 1 & 2 & 3 & 4 & 5 & 6 & 7 & 8 & 9 & 0 & 1 & 2 & 3 & 4 & 5 & 6 & 7 & 8 & 9 & 0 & 1 & 2 & 3 & 4 & 5\end{array}$

Fig.2 The initial network diagram of multi-project

As can be seen from figure 2, the critical path of project A is (A1-A2-A3-A4-A6-A8-A9-A10), the duration of project $\mathrm{A}$ is 35 weeks; the critical path of project $\mathrm{B}$ is (B-B1-B2-B5-B7-B8-B9), the duration of project $\mathrm{B}$ is 29 weeks; the critical path of project $\mathrm{C}$ is $(\mathrm{C} 1-\mathrm{C} 5-\mathrm{C} 7-\mathrm{C} 8-\mathrm{C} 9)$, the duration of project $\mathrm{C}$ is 33 weeks.

The mathematical model of the problem is shown as follows:

$$
\begin{gathered}
\lambda_{1}=\lambda_{2}=0.5, c_{1}=0.6, c_{2}=0.4 \\
M A X . E V=0.5 * \sum_{w=1}^{2} c_{w} * E V_{K}^{w}+\lambda_{2} * \frac{1}{3} * \sum_{l=1}^{3} \sum_{w=1}^{2} c_{w} * E V_{l}^{w}
\end{gathered}
$$




$$
\begin{aligned}
& \text { MAX.EV }=\frac{1}{2} * \sum_{w=1}^{2} c_{w} * \sum_{\mathrm{t}=1}^{41}\left[\left\{R^{w}(t) / \sum_{t=1}^{T} R^{w}(t)\right\}^{*} \ln \left\{R^{w}(t) / \sum_{t=1}^{T} R^{w}(t)\right\}\right] \\
& +\frac{1}{6} * \sum_{l=1}^{3} \sum_{w=1}^{2} c_{w} * \sum_{\mathrm{t}=1}^{T_{l}}\left[\left\{R_{l}^{w}(t) / \sum_{t=1}^{T_{l}} R_{l}^{w}(t)\right\}^{*} \ln \left\{R_{l}^{w}(t) / \sum_{t=1}^{T_{l}} R_{l}^{w}(t)\right\}\right]
\end{aligned}
$$

The priority relationship is expressed as a penalty function, which is reflected in the fitness function, that is, when the genetic operation is performed. The non-feasible solution will be eliminated due to the penalty. The fitness function is shown as follows:

$$
\begin{gathered}
\text { fit }=M+\frac{1}{2} * \sum_{m=1}^{2} c_{m} * \sum_{\mathrm{t}=1}^{25}\left[\left\{R^{m}(t) / \sum_{t=1}^{T} R^{m}(t)\right\}^{*} \ln \left\{R^{m}(t) / \sum_{t=1}^{T} R^{m}(t)\right\}\right] \\
+\frac{1}{6} * \sum_{l=1}^{3} \sum_{m=1}^{2} c^{m} * \sum_{\mathrm{t}=1}^{T_{l}}\left[\left\{R_{l}^{m}(t) / \sum_{t=1}^{T_{l}} R_{l}^{m}(t)\right\} * \ln \left\{R_{l}^{m}(t) / \sum_{t=1}^{T_{l}} R_{l}^{m}(t)\right\}\right]-\alpha f(x) \\
\alpha f(x)=\max \left(T_{S}\left(B_{4}\right)+4-T_{S}\left(B_{6}\right), T_{S}\left(C_{2}\right)+7-T_{S}\left(C_{3}\right), T_{S}\left(C_{2}\right)+7-T_{S}\left(C_{4}\right), 0\right)
\end{gathered}
$$

In this paper, we use the single point crossover operator and the basic consistency mutation operator to select the operator by roulette method. The termination condition is the number of iteration come to 300 times. In the test, the genetic parameters are as follows:

$$
M=2000 ; \quad \varphi=400 ; \quad P_{c}=0.8 ; P_{m}=0.02
$$

After about 100 iterations, the optimal solution fitness value is obtained fit $=2003.47$. The actual start time for each non-critical chain activity is shown as follows:

$$
\begin{aligned}
& T_{S}\left(A_{5}\right)=21 ; T_{S}\left(A_{7}\right)=33 ; T_{S}\left(B_{3}\right)=17 ; T_{S}\left(B_{4}\right)=21 ; T_{S}\left(B_{6}\right)=25 ; \\
& T_{S}\left(C_{2}\right)=3 ; T_{S}\left(C_{3}\right)=T_{S}\left(C_{4}\right)=10 ;
\end{aligned}
$$

The maximum average resource requirement of $\mathrm{C} 1$ was 36 before optimization. After the optimization, the maximum average resource requirement of $\mathrm{C} 1$ is 28 ; the maximum average resource requirement of $\mathrm{C} 2$ was 18 before the optimization, and after the optimization, the maximum average of $\mathrm{C} 2$ is 14 , we can see that the resource equilibrium model has achieved some effect.

\section{Summary}

In this paper, through the analysis of the existing resource equilibrium models, a resource leveling optimization model of multi-project based on entropy function is adopted. And the advantages and disadvantages of different methods of resource leveling optimization are analyzed. At the same time, the concept of resource entropy is introduced to improve the existing single project model, and a new multi-project resource equilibrium model based on resource entropy is proposed. Finally, considering the superiority of intelligent algorithms, this paper proposes a genetic algorithm to solve the model.

\section{Acknowledgments}

This paper is sponsored by National Natural Science Foundation of China (GN: 71271105) and Humanities and Social Sciences Planning Fund of Ministry of Education (GN: 12YJA630036).

\section{References}

[1] Gong J, Weng L, Liu S. An improved ant colony optimization algorithm for nonlinear resource-leveling problems [J]. Computers and Mathematics with Application, 201 1, 61 (8): 2300-2305. (In Chinese). 
[2] MENG Xiang hai, ZHENG Lai, QIN Guanming. Traffic accidents prediction and prominent influencing factors analysis based on fuzzy logic [J]. Journal of Transportation Systems Engineering and Information Technology. 2009, (2): 87-92. (In Chinese).

[3] GAO Dezhi, LI Wei,DUAN Jianmin, ZHENG Banggui. Lane Detection Using Fuzzy Logic [J]. Journal of Beijing University of Technology, 2011, (7):971-977. (In Chinese).

[4] Anagnostopoulos K P, Koulinas G K. A simulated annealing hyper-heuristic for construction resource leveling [J]. Construction Management \& Economics, 2010, 28(2): 163-175.

[5] QI Jianxun, WANG Qiang, JIA Haihong. Research on New Methods Based on Entropy and Particle Swarm Optimization for Resource Leveling Problem [J]. Chinese Journal of Management Science, 2008, 16(1): 90-95. (In Chinese).

[6] Megow N, Mohring R H, Schulz J. Decision support and "optimization in shutdown and turnaround scheduling [J]. INFORMS J on Computing, 2011, 23(2): 189-204.

[7] Li H B, Xu Z. Robust project scheduling: A review [J]. Systems Engineering, 2014, 32(2): 123-131. (In Chinese).

[8] Christodoulou S, Ellinas G, Aslani P. Entropy-based scheduling of resource-constrained construction projects [J]. Automation in Construction, 2009, 18(7): 919-928.

[9] He L, Zhang L. Dynamic priority rule-based forward backward heuristic algorithm for resource leveling problem in construction project [J]. J of the Operational Research Society, 2013, 64(8): 1106-1117.

[10] Tang Y, Liu R, Sun Q. Two-stage scheduling model for resource leveling of linear projects [J]. Journal of Construction Engineering and Management, 2014, 140(7): 1-10.

[11] Pang N S, Ji C M. Leveling optimization for multi-mode and discrete resource under generalized precedence relations [J]. J of Systems Engineering, 2011, 26(4): 538-545. (In Chinese).

[12] Xie J R, Liu C X, Zhou Y H. The Hop field-based solution for resource-leveling problem [J]. Systems Engineering Theory \& Practice, 2006, 26(3): 83-87. (In Chinese).

[13] Pang N S, Ji C M, Qi J X. Leveling optimization of parallel adjustment to resource divided period and work translation based on modified differential evolution $[\mathrm{J}]$. Chinese $\mathrm{J}$ of Management Science, 2009, 17(6): 130-138. (In Chinese).

[14] Song Y J, Ye C M, Huang Z Y. Cuckoo search algorithm for multi-resource leveling optimization [J]. J of Computer Applications, 2014, 34(1): 189-193. (In Chinese).

[15] Kyriklidis C, Vassiliadis V, Kirytopoulos K, et al. Hybrid nature-inspired intelligence for the resource leveling problem [J]. Int J of Operational Research, 2014, 14(3): 387-407.

[16] Afshar-Nadjafi B, Najjarbashi H, Mehdizadeh E. A branch-and-bound procedure for resource leveling in multi-mode resource constraint project scheduling problem [J]. Research J of Recent Sciences, 2012, 1(7): 33-38.

[17] Coughlan E T, Lubbecke M E, Schulz J. A branch-price algorithm for multi-mode resource leveling [C]. Experimental Algorithms. Berlin Heidelberg: Springer, 2010: 226-238.

[18] Hariga M, El-Sayegh S M. Cost optimization model for the multi-resource leveling problem with allowed activity splitting [J]. J of Construction Engineering and Management, 2011, 137(1): 56-64.

[19] Guo Y, Li N, Li X S. Multi-mode multiple resources leveling and multi-objective particle 
swarm optimization with dynamic population [J]. Control and Decision, 2013, 28 (1): 131-136. (In Chinese).

[20] Ashuri B, Tavakolan M. Fuzzy enabled hybrid genetic algorithm - Particle swarm optimization approach to solve TCRO problems in construction project planning [J]. J of Construction Engineering and Management, 2012,138 (9): 1065-1074. 